\title{
A LARGE C+N+O ABUNDANCE SPREAD IN GIANT STARS OF THE GLOBULAR CLUSTER NGC 1851*
}

\author{
David Yong ${ }^{1}$, Frank Grundahl ${ }^{2}$, Francesca D’Antona ${ }^{3}$, Amanda I. Karakas ${ }^{1}$, John C. Lattanzio ${ }^{4}$, and John E. Norkis ${ }^{1}$ \\ ${ }^{1}$ Research School of Astronomy and Astrophysics, Australian National University, Weston, ACT 2611, Australia; yong@mso.anu.edu.au, \\ akarakas@mso.anu.edu.au,jen@mso.anu.edu.au \\ ${ }^{2}$ Department of Physics and Astronomy, Aarhus University, Denmark; fgj@phys.au.dk \\ ${ }^{3}$ INAF-Osservatorio Astronomico di Roma, via Frascati 33, 00040 Monteporzio, Italy; dantona@mporzio.astro.it

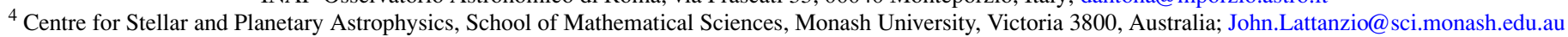 \\ Received 2008 December 19; accepted 2009 February 10; published 2009 March 23
}

\begin{abstract}
Abundances of $\mathrm{C}, \mathrm{N}$, and $\mathrm{O}$ are determined in four bright red giants that span the known abundance range for light $(\mathrm{Na}$ and $\mathrm{Al}$ ) and $s$-process $(\mathrm{Zr}$ and $\mathrm{La}$ ) elements in the globular cluster $\mathrm{NGC} 1851$. The abundance sum $\mathrm{C}+\mathrm{N}+\mathrm{O}$ exhibits a range of 0.6 dex, a factor of 4 , in contrast to other clusters in which no significant $\mathrm{C}+\mathrm{N}+\mathrm{O}$ spread is found. Such an abundance range offers support for the Cassisi et al. scenario in which the double subgiant branch populations are coeval but with different mixtures of $\mathrm{C}+\mathrm{N}+\mathrm{O}$ abundances. Further, the $\mathrm{Na}, \mathrm{Al}, \mathrm{Zr}$, and La abundances are correlated with $\mathrm{C}+\mathrm{N}+\mathrm{O}$, and therefore NGC 1851 is the first cluster to provide strong support for the scenario in which asymptotic giant branch stars are responsible for the globular cluster light element abundance variations.
\end{abstract}

Key words: Galaxy: abundances - globular clusters: individual (NGC 1851) - stars: abundances

\section{INTRODUCTION}

Globular clusters have long been regarded as simple stellar populations which may be described by a single age, helium abundance $(Y)$, metallicity $(Z)$, and initial mass function (Renzini \& Buzzoni 1986). For many years, these simple stellar populations have played a prominent role in astronomy by providing a lower limit to the age of the universe and for testing the predictions of stellar evolution and stellar nucleosynthesis (e.g., see review by Gratton et al. 2004).

However, a revolution in the field of globular cluster research is underway, prompted by the recent discoveries of complex structure on the main sequence, subgiant branch (SGB), red giant branch (RGB), and/or horizontal branch (HB) within some Galactic and extra-Galactic globular clusters indicating that these simple stellar populations in fact contain discrete, multiple populations (e.g., Bedin et al. 2004; D’Antona et al. 2005; Piotto et al. 2005; Sollima et al. 2005; Mackey et al. 2008). At present, these multiple populations can be best explained in terms of distinct ages and/or compositions, although the sequence of events leading to their formation remains largely unexplained (Renzini 2008). In some cases, extremely large helium abundances, up to $Y=0.40$, are required to explain the various populations (e.g., D'Antona \& Caloi 2004; Norris 2004). While theoretical models have struggled to account for such high abundances (e.g., Karakas et al. 2006a; Bekki \& Chiba 2007), some successful efforts are beginning to appear (e.g., D'Ercole et al. 2008; Pumo et al. 2008). The intrigue surrounding the multiple populations in globular clusters has been enhanced by the speculation that clusters with extended HBs, generally the most massive clusters, are the remnants of dwarf galaxies from which the halo of the Galaxy was built (Lee et al. 2007).

The globular cluster NGC 1851 has a bimodal HB and displays multiple SGBs (Milone et al. 2008, hereafter M08), but has no evidence for multiple main sequences. Based on the width of the main sequence and RGB, M08 suggested that the maximum possible abundance variation is $\Delta[\mathrm{Fe} / \mathrm{H}]=0.1 \mathrm{dex}$ or $\Delta Y=0.026$. From an analysis of eight bright RGB stars

\footnotetext{
* This paper includes data gathered with the 6.5 meter Magellan Telescopes located at Las Campanas Observatory, Chile.
}

using high-resolution spectroscopy, Yong \& Grundahl (2008, hereafter YG08) showed that the dispersion in $[\mathrm{Fe} / \mathrm{H}]$ was less than $0.1 \mathrm{dex}$, and that NGC 1851 displays the usual star-tostar abundance variations for the light elements $\mathrm{O}, \mathrm{Na}$, and $\mathrm{Al}$; the exact mechanism for these abundance patterns, seen in all globular clusters, remains unknown (e.g., Gratton et al. 2004). However, YG08 also showed that NGC 1851 harbors a star-tostar abundance variation for the $s$-process elements $\mathrm{Zr}$ and $\mathrm{La}$ and that the abundances of these elements were correlated with the light element abundances. Furthermore, within the small sample there was a hint that the abundances of the $s$-process elements was bimodal, which suggests that the RGB may consist of two populations with distinct chemical compositions. Therefore, as well as showing unusual photometric properties, NGC 1851 also displays peculiar chemical abundance patterns not seen in other clusters.

Several explanations for the double SGB in NGC 1851 have been offered. If age is the sole parameter, M08 suggested a $1 \mathrm{Gyr}$ difference with the fainter subgiant branch (fSGB) population being older. M08 also considered a combination of increasing $[\mathrm{Fe} / \mathrm{H}]$ by 0.2 dex and helium from $Y=0.247$ to 0.30 , though this possibility was excluded by the magnitudes of the blue $\mathrm{HB}$ stars. An alternate explanation was proposed by Cassisi et al. (2008) in which the two SGB populations are coeval, but with the fSGB population having a total $\mathrm{C}+\mathrm{N}+\mathrm{O}$ abundance increased by a factor of 2 . In this scenario, the two populations may have comparable He abundance (at most $\Delta Y=0.032$ ), in contrast to the large He variations inferred in other clusters which display multiple populations. We have obtained spectra with the goal of measuring the abundance sum $\mathrm{C}+\mathrm{N}+\mathrm{O}$ to test the Cassisi et al. (2008) scenario.

\section{OBSERVATIONS AND ABUNDANCE ANALYSIS}

We selected four bright RGB stars from YG08, two Na-, $\mathrm{Al}-, \mathrm{Zr}-$, and La-normal targets as well as two Na-, Al-, Zr-, and La-rich objects. In Figure 1, we show the locations of our targets in a color-magnitude diagram. If the double SGB populations are due to distinct chemical compositions, and if these chemical compositions are also present on the RGB, then 


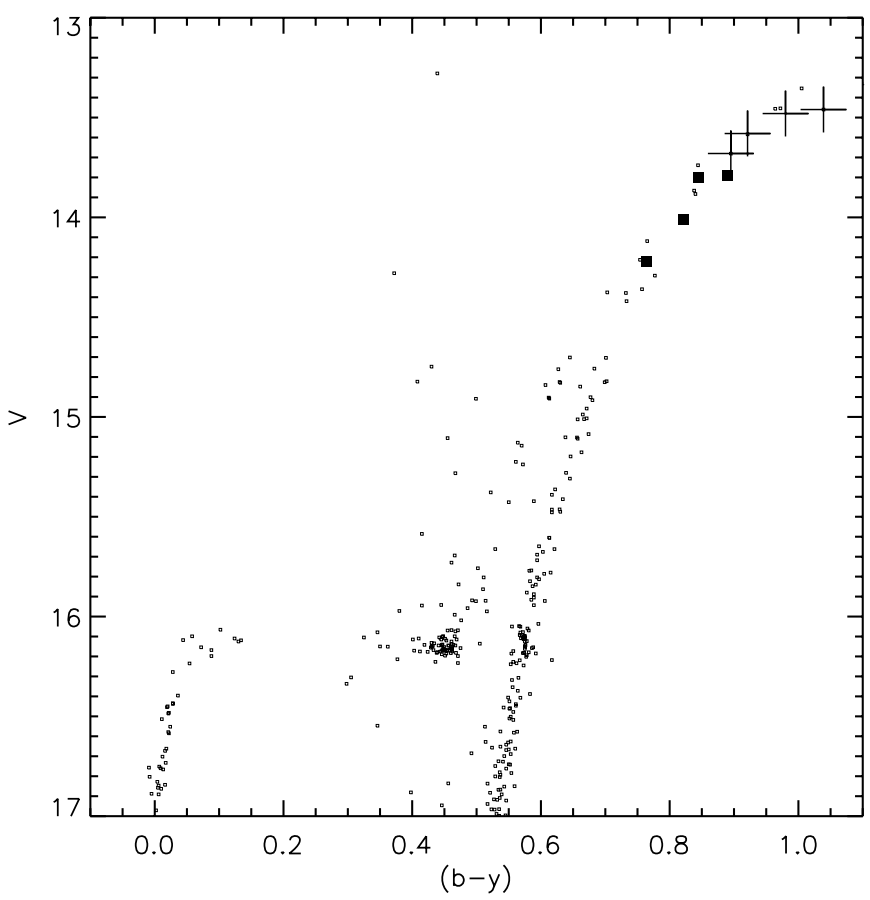

Figure 1. Color-magnitude diagram for NGC 1851 using the Grundahl et al. (1999) photometry. The large plus symbols represent the bright giants in this study. The filled squares represent the additional four stars in YG08.

our small, but carefully selected, sample should allow us to identify any $\mathrm{C}+\mathrm{N}+\mathrm{O}$ abundance variation, if present.

The stars were observed with the high-resolution spectrograph MIKE (Bernstein et al. 2003) on the Magellan Clay Telescope on 2007 December 23. We used the 0.'7 slit which provided a spectral resolution of $R \simeq 37,000$ in the blue CCD (3350-5000 $\AA$ ) and $R \simeq 30,000$ in the red CCD (4900-9500 $)$ as measured from the ThAr lines. Our total exposure times per star ranged from 40 to 80 minutes resulting in signal-to-noise ratios (S/N) per four pixel resolution element of 70-100 at $4320 \AA$ and 210-260 at $6600 \AA$. The data were reduced using the same procedure described by Yong et al. (2006).

Although the new data are of lower spectral resolution $(R \simeq 30,000$ vs. $R \simeq 55,000)$, the $\mathrm{S} / \mathrm{N}$ and wavelength coverage are superior to the spectra analyzed by YG08. Using these new spectra, we rederived the stellar parameters using the same tools and techniques described in YG08 and found that the revised stellar parameters are in good agreement with the stellar parameters previously obtained. The new dispersion in $\mathrm{Fe}$ abundances for these four stars is $\sigma[\mathrm{Fe} / \mathrm{H}]=0.03 \mathrm{dex}$ compared with 0.11 dex for the YG08 analysis. This decrease is due to the increased wavelength coverage and $\mathrm{S} / \mathrm{N}$ which allows us to more accurately measure the equivalent widths of a larger number of $\mathrm{Fe}$ lines.

The abundances of $\mathrm{C}$ and $\mathrm{N}$ were derived by comparing observed spectra with synthetic spectra generated using the LTE line analysis and spectrum synthesis program MOOG (Sneden $1973)$. For C, we analyzed lines from the $(0,0)$ and $(1,1)$ bands of the $A-X$ electronic transition of the $\mathrm{CH}$ molecule near 4310 $\AA$ using the line list from Plez et al. (2008). For N, we analyzed lines from the $(2,0)$ band of the $A-X$ electronic transition of the $\mathrm{CN}$ molecule near $8000 \AA$ using the line list from Reddy et al. (2002) which is based on the de Laverny \& Gustafsson (1998) list. In both cases, we adjusted the abundances of $\mathrm{C}$ or $\mathrm{N}$ until the synthetic spectra matched the observed spectra (see Figures 2
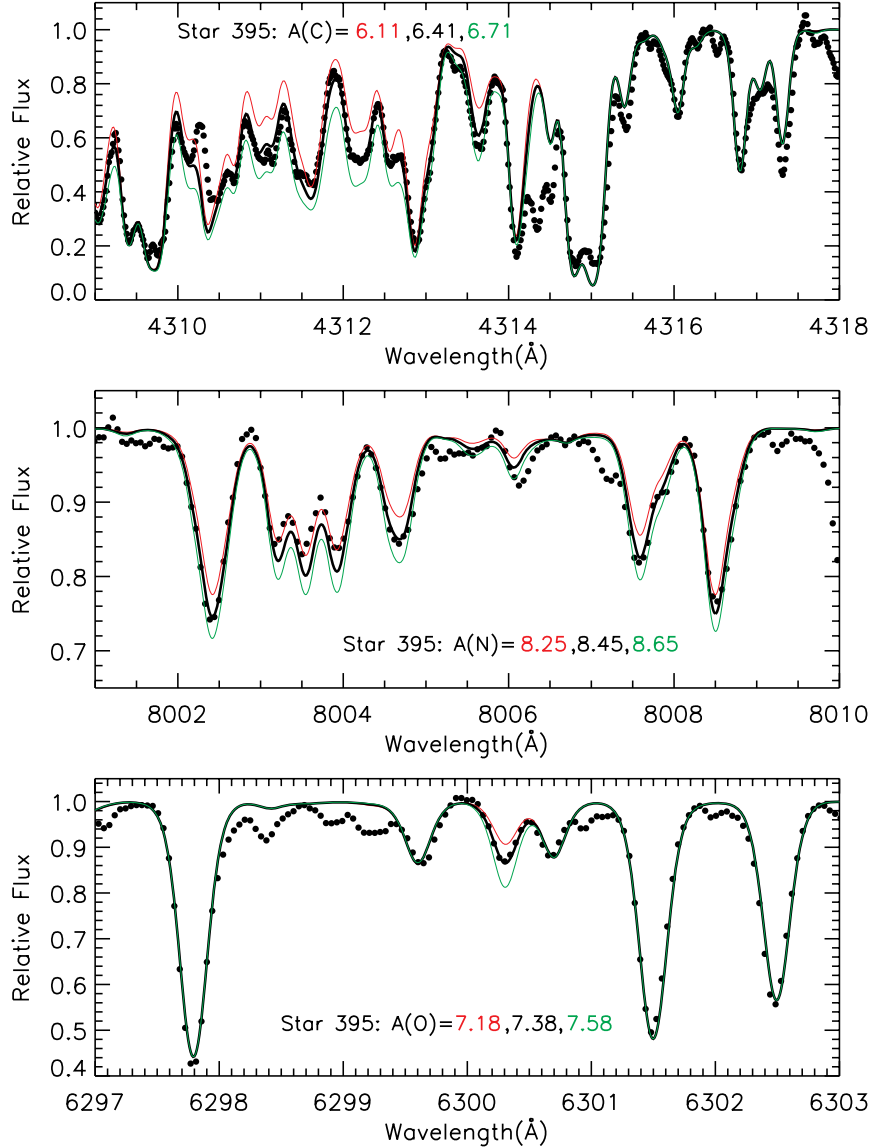

Figure 2. Observed spectra (circles) and synthetic spectra (lines) for C (upper), $\mathrm{N}$ (middle), and $\mathrm{O}$ (lower) for Star 395. The synthetic spectra show the best fit (thick black line) and unsatisfactory fits (thin red and green lines) $A(\mathrm{C}) \pm 0.30$ $\operatorname{dex}, A(\mathrm{~N}) \pm 0.20 \mathrm{dex}$, and $A(\mathrm{O}) \pm 0.20 \mathrm{dex}$.

and 3). We rederived the $\mathrm{O}$ abundances again from the $6300 \AA$ $[\mathrm{O}$ I] line using the new spectra, the revised $\mathrm{C}$ and $\mathrm{N}$ abundances, and the revised stellar parameters. Since the abundances of C, N, and $\mathrm{O}$ are coupled, we iterated until self-consistent abundances were obtained. We also updated the abundances for $\mathrm{Na}, \mathrm{Al}, \mathrm{Zr}$, and La using the new spectra and revised stellar parameters (a complete abundance analysis of our data will be presented elsewhere). We present the stellar parameters and abundances in Table 1.

\section{RESULTS}

As mentioned, our sample was selected to span the full range of the known star-to-star abundance variation of $\mathrm{Na}, \mathrm{Al}, \mathrm{Zr}$, and La. Our new analysis confirms the large abundance spread for these elements as well as the correlations between the light $(\mathrm{Na}$ and $\mathrm{Al}$ ) and heavy ( $\mathrm{Zr}$ and La) elements established by YG08. The range in $\mathrm{Na}, \mathrm{Al}, \mathrm{Zr}$, and $\mathrm{La}$ abundances far exceeds the measurement uncertainties of $0.06,0.05,0.12$, and $0.08 \mathrm{dex}$, respectively, though we note that the abundance amplitudes for these elements are slightly smaller than those reported by YG08.

Adopting a solar abundance $\log \epsilon(\mathrm{Fe})=7.50$ (Grevesse \& Sauval 1998), we find a mean cluster abundance of $[\mathrm{Fe} / \mathrm{H}]=$ $-1.22(\sigma=0.03)$. The revised cluster Fe abundance is in good agreement with the YG08 value and previous estimates in the literature, but the abundance dispersion is considerably smaller for the reasons noted above. The abundance range for Fe may be entirely attributable to observational uncertainties (0.07 dex). 
Table 1

Stellar Parameters and Abundances

\begin{tabular}{cccccccccccccc}
\hline \hline Star $^{\mathrm{a}}$ & $V$ & $T_{\text {eff }}(\mathrm{K})$ & $\log g(\mathrm{cgs})$ & $v_{t}\left(\mathrm{~km} \mathrm{~s}^{-1}\right)$ & $A(\mathrm{Fe})$ & $A(\mathrm{C})$ & $A(\mathrm{~N})$ & $A(\mathrm{O})$ & $A(\mathrm{CNO})$ & $A(\mathrm{Na})$ & $A(\mathrm{Al})$ & $A(\mathrm{Zr})$ & $A(\mathrm{La})$ \\
\hline 003 & 13.68 & 4125 & 0.7 & 1.86 & 6.28 & 6.46 & 7.60 & 7.75 & 8.00 & 5.25 & 5.63 & 1.51 & 0.16 \\
095 & 13.58 & 4125 & 0.7 & 1.92 & 6.32 & 6.51 & 7.60 & 7.60 & 7.92 & 5.25 & 5.69 & 1.64 & 0.22 \\
329 & 13.46 & 3925 & 0.0 & 1.92 & 6.25 & 6.81 & 8.00 & 7.88 & 8.26 & 5.50 & 5.79 & 1.87 & 0.42 \\
395 & 13.48 & 4025 & 0.5 & 2.01 & 6.29 & 6.41 & 8.45 & 7.38 & 8.49 & 5.74 & 6.04 & 1.85 & 0.53 \\
\hline
\end{tabular}

Note.

${ }^{\text {a }}$ Star names taken from Stetson (1981).
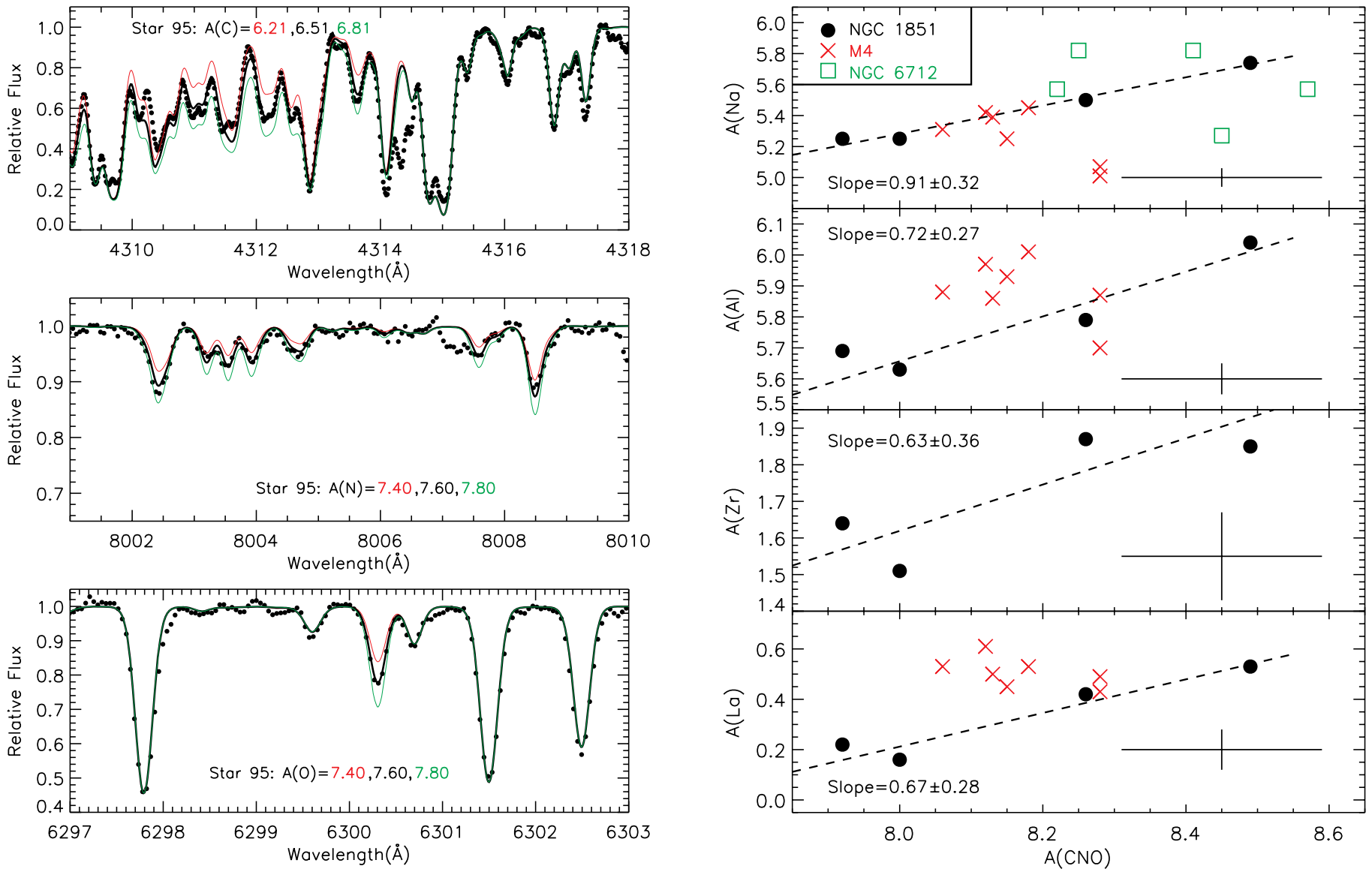

Figure 3. Same as Figure 2 but for Star 95

For $\mathrm{C}$ and $\mathrm{N}$, we find a large range in abundances. The 0.40 dex range in $\log \epsilon(\mathrm{C})$ and the 0.85 dex range in $\log \epsilon(\mathrm{N})$ greatly exceed the measurement uncertainties $(0.04$ dex and 0.11 dex, respectively). The abundance amplitude for $\mathrm{N}, \Delta \log \epsilon(\mathrm{N})=$ 0.85 , is smaller than that seen in other clusters, e.g., RGB bump stars in NGC 6752 and main-sequence stars in M13 show 2.0 dex amplitudes (Briley et al. 2002; Yong et al. 2008a). This may be due to the small sample and/or advanced evolutionary status where extensive $\mathrm{CN}$ cycling may reduce the amplitude of the $\mathrm{N}$ abundance dispersion. Curiously, we do not detect any obvious anticorrelation between $\mathrm{C}$ and $\mathrm{N}$ as seen in every well studied Galactic globular cluster. We identify correlations between the abundances of $\mathrm{N}$ and $\mathrm{Na}$ as well as $\mathrm{N}$ and $\mathrm{Al}$, but the corresponding anticorrelations between $\mathrm{C}$ and $\mathrm{Na}$ and $\mathrm{C}$ and $\mathrm{Al}$ are not obvious. Nevertheless, we find correlations between the abundances of $\mathrm{N}$ and $\mathrm{Zr}$ as well as $\mathrm{N}$ and $\mathrm{La}$ as expected given the known behavior of $\mathrm{N}$ and $\mathrm{Al}$ and the correlations between $\mathrm{Al}$ and $\mathrm{Zr}$ and $\mathrm{Al}$ and $\mathrm{La}$ found in $\mathrm{YG} 08$.

Figure 4. Abundances of $\mathrm{Na}, \mathrm{Al}, \mathrm{Zr}$, and $\mathrm{La}$ vs. the abundance sum $\mathrm{C}+\mathrm{N}+\mathrm{O}$ A representative error bar and a linear least-squares fit to the data (including formal slope and error) are shown. Data for M4 (Smith et al. 2005) and NGC 6712 (Yong et al. 2008b) are included for comparison.

Our first significant result is that the abundance sum $\mathrm{C}+\mathrm{N}+\mathrm{O}$ shows a large variation. Within our small but biased sample, the amplitude of the abundance variation is $\Delta A(\mathrm{C}+\mathrm{N}+\mathrm{O})=0.57$ dex. The 0.57 dex spread far exceeds the estimated uncertainty of 0.14 dex. Bright giants in M4 and NGC 6712, globular clusters of comparable metallicity to NGC 1851 , show $\mathrm{C}+\mathrm{N}+\mathrm{O}$ abundance amplitudes of 0.22 dex and 0.35 dex, respectively (Smith et al. 2005; Yong et al. 2008b). Unevolved stars in NGC 6397, NGC 6752, and 47 Tuc show $\mathrm{C}+\mathrm{N}+\mathrm{O}$ abundances that may be regarded as constant within the $\sim 0.3$ dex uncertainties (Carretta et al. 2005).

Our second main result is that the abundances of $\mathrm{Na}, \mathrm{Al}$, $\mathrm{Zr}$, and La show a positive correlation with the abundance sum $\mathrm{C}+\mathrm{N}+\mathrm{O}$ (see Figure 4). In this figure, we overplot results for M4 and NGC 6712 and find that for the available data there 
are no positive correlations between $A(X)$ and $\mathrm{C}+\mathrm{N}+\mathrm{O}$ in these other clusters. Finally, our small sample does not exclude the possibility that the $\mathrm{C}+\mathrm{N}+\mathrm{O}$ abundance may be bimodal within this cluster.

\section{DISCUSSION}

We seek to test the Cassisi et al. (2008) prediction in which the double SGB in NGC 1851 is due to two coeval populations whose chemical compositions for $\mathrm{C}+\mathrm{N}+\mathrm{O}$ differ by a factor of 2. Note that we have not directly observed stars on the SGB. Instead, we have observed a sample of bright RGB stars in which we have found a large spread in the $\mathrm{C}+\mathrm{N}+\mathrm{O}$ abundances. If the SGBs comprise populations with different $\mathrm{C}+\mathrm{N}+\mathrm{O}$ abundances, then we assume that these populations with distinct compositions are also present on the main sequence and RGB. Strömgren photometry obtained by Grundahl et al. (1999) reveals that the bimodality seen on the SGB can also be found on the RGB. Therefore, the large spread in $\mathrm{C}+\mathrm{N}+\mathrm{O}$ abundances in RGB stars offers support, albeit not definitive, for the Cassisi et al. (2008) scenario. We tentatively confirm that the double SGB in NGC 1851 is due to two coeval populations with $\mathrm{C}+\mathrm{N}+\mathrm{O}$ abundances differing by a factor of 2 .

Based on the relative numbers of stars, YG08 speculated that the brighter subgiant branch (bSGB) stars were related to the RGB stars with "normal" $\mathrm{Zr}$ and $\mathrm{La}$ abundances and that these stars populate the red HB. YG08 also suggested that the fSGB stars are related to the RGB stars with $\mathrm{Zr}$ and La enhancements and that these stars populate the blue HB. If the fSGB is older than the bSGB as M08 suggest, then the YG08 speculation would require the $\mathrm{Zr}$-rich, La-rich stars to be older than the Zr-normal, La-normal stars. In this scenario, the sequence of events leading to cluster formation would be complicated and require the asymptotic giant branch (AGB) or other stars to preferentially pollute the older Zr-rich La-rich population but without polluting the gas from which the younger $\mathrm{Zr}$-normal La-normal population formed. However, if the $\mathrm{C}+\mathrm{N}+\mathrm{O}$ abundance of the $s$-process-rich stars is larger than that of the $s$-process-normal ones, the fSGB may be coeval to the bSGB, or even a bit younger than the bSGB, as shown in Cassisi et al. (2008). Due to the observational errors, and to the fact that the age difference we are looking for does not exceed some $100 \mathrm{Myr}$, it may be very difficult or impossible to constrain the precise mass range of the progenitors of the $s$-process-rich stars, but a coherent picture of the chemical evolution history is emerging.

The Cassisi et al. (2008) scenario has important implications for other clusters which display multiple SGBs. Villanova et al. (2007) studied the SGB region of $\omega$ Centauri, the most massive globular cluster which is suspected of harboring up to five populations with discrete ages and/or compositions. From the large range in the magnitudes of the SGB populations, Villanova et al. (2007) inferred a large range in ages. They found that there was an extremely old, but metal-rich population which greatly complicates any formation scenario. Based on our NGC 1851 results, the inferred ages of SGB stars in $\omega$ Cen requires detailed knowledge of the relative abundances of $\mathrm{C}+\mathrm{N}+\mathrm{O}$. We speculate that the old metal-rich population (Villanova et al.'s "SGB group D") has a large excess of $[(\mathrm{C}+\mathrm{N}+\mathrm{O}) / \mathrm{Fe}]$ relative to the more metal-poor populations and therefore a younger relative age than currently inferred. Such compositions would be consistent with our understanding of the composition of the ejecta from AGB stars (Karakas \& Lattanzio 2007) and the prominent role of AGB stars in the chemical evolution of $\omega$ Cen (Norris \& Da Costa 1995).

AGB stars have long been suspected as being responsible, in part or in whole, for the star-to-star abundance variation for the light elements $(\mathrm{C}, \mathrm{N}, \mathrm{O}, \mathrm{F}, \mathrm{Na}, \mathrm{Mg}$, and $\mathrm{Al})$ found in every well studied Galactic globular cluster, including NGC 1851. However, various theoretical yields and chemical evolution models involving AGB stars have yet to provide a satisfactory solution (e.g., Fenner et al. 2004). AGB stars may be expected to produce a substantial increase in the $\mathrm{C}+\mathrm{N}+\mathrm{O}$ abundance as they enhance $\mathrm{Na}$ and $\mathrm{Al}$ and deplete $\mathrm{O}$ and $\mathrm{Mg}$. AGB stars should also produce $s$-process elements, albeit via different processes than for the $\mathrm{O}, \mathrm{Na}, \mathrm{Mg}$, and $\mathrm{Al}$ nucleosynthesis. If AGB stars are responsible for the light element abundance variations in globular clusters, we may expect a large range in $\mathrm{C}+\mathrm{N}+\mathrm{O}$ abundances along with correlations between (1) $\mathrm{C}+\mathrm{N}+\mathrm{O}$ and $\mathrm{Na}$, (2) $\mathrm{C}+\mathrm{N}+\mathrm{O}$ and $\mathrm{Al}$, and (3) $\mathrm{C}+\mathrm{N}+\mathrm{O}$ and $s$-process elements. On the other hand, these proposed abundance trends are dependent on the number of third dredge-up episodes and therefore on the adopted convection efficiency (e.g., Ventura \& D'Antona 2005a; Renzini 2008) and mass loss (e.g., Ventura \& D'Antona 2005b). Further uncertainty in the yields comes from the uncertainties in the nuclear reaction rates (e.g., Lugaro et al. 2004; Ventura \& D’Antona 2006; Karakas et al. 2006b; Izzard et al. 2007). Although the brightest AGB stars in the LMC and SMC can become C-rich, it is possible that nucleosynthesis in the most massive AGBs is scarcely affected by the third dredge-up, with only a minor increase in the total $\mathrm{C}+\mathrm{N}+\mathrm{O}$ abundance of the second generation stars (D'Antona \& Ventura 2008). Nevertheless, the absence of the signatures listed above has forced astronomers to consider alternate sources such as massive stars (e.g., Decressin et al. 2007).

The correlations between $\mathrm{C}+\mathrm{N}+\mathrm{O}$ and $\mathrm{Na}, \mathrm{Al}, \mathrm{Zr}$, and La presented here offer strong support for the scenario in which AGB stars are responsible for the light element abundance variations. Given the peculiar photometric and chemical properties of NGC 1851 , it is surprising that this cluster offers the strongest support for the globular cluster AGB pollution scenario. It would be ironic if NGC 1851 is the only globular cluster for which the AGB pollution scenario is applicable. An alternate interpretation is that the AGB pollution scenario is applicable to all globular clusters. For NGC 1851, the masses of the polluting AGB stars were lower such that the products of third dredgeup, increased $\mathrm{C}+\mathrm{N}+\mathrm{O}$ and $s$-process elements, are clearly evident.

We note that abundance spreads in $A(\mathrm{CNO})$ are likely present in $\omega$ Centauri (Norris \& Da Costa 1995), although they were concerned with possible problems with the $\mathrm{N}$ measurements. Indeed, NGC 1851 bears a striking resemblance to $\omega$ Centauri. Both clusters possess multiple SGBs, large abundance spreads for $s$-process elements and likely spreads in $A(\mathrm{CNO})$, and a scatter in the Strömgren $m_{1}$ index, traditionally used as a metallicity indicator (Grundahl et al. 1999). We reiterate the comments by YG08 that NGC 1851 is a "bridge" between $\omega$ Centauri (variation of all elements) and NGC 6752-like clusters (constant $\mathrm{Fe}$ and $\mathrm{C}+\mathrm{N}+\mathrm{O}$ but large variations of light elements C-Al).

Finally, recent work by Salaris et al. (2008) also suggests a relationship between the SGB and HB populations in NGC 1851. Specifically, the bSGB stars evolve onto the red HB, while the fSGB stars populate the red and blue HBs. If this is correct, we would therefore expect stars on the red $\mathrm{HB}$ to be primarily Zr-normal and La-normal. NGC 1851 may provide critical 
information on HB morphology and the second parameter effect and additional studies of this cluster are of great interest.

D.Y. thanks Bertrand Plez for providing the $\mathrm{CH}$ line list and Bacham Reddy for providing the $\mathrm{CN}$ line list. We thank the anonymous referee for helpful comments. F.G. acknowledges financial support from the Carlsberg Foundation, the Danish AsteroSeismology Centre at Aarhus University, and support from the Danish National Research Council to the project: "Stars: Central engines of the evolution of the universe." F.D. acknowledges partial support from the Italian PRIN MIUR 2007 "Multiple stellar populations in globular clusters: census, characterization and origin." We acknowledge support from the Australian Research Council's Discovery Projects funding scheme under grants DP0663562 (J.E.N.) and DP0664105 (A.I.K.). D.Y. acknowledges financial support from the Access to Major Research Facilities Programme which is a component of the International Science Linkages Programme established under the Australian Government's innovation statement, Backing Australia's Ability. Australian access to the Magellan Telescopes was supported through the Major National Research Facilities program of the Australian Federal Government.

\section{REFERENCES}

Bedin, L. R., Piotto, G., Anderson, J., Cassisi, S., King, I. R., Momany, Y., \& Carraro, G. 2004, ApJ, 605, L125

Bekki, K., \& Chiba, M. 2007, ApJ, 665, 1164

Bernstein, R., Shectman, S. A., Gunnels, S. M., Mochnacki, S., \& Athey, A. E. 2003, in Proc. SPIE 4841, ed. M. Iye \& A. F. M. Moorwood (Bellingham, WA: SPIE) 1694

Briley, M. M., Cohen, J. G., \& Stetson, P. B. 2002, ApJ, 579, L17

Carretta, E., Gratton, R. G., Lucatello, S., Bragaglia, A., \& Bonifacio, P. 2005, A\&A, 433, 597

Cassisi, S., Salaris, M., Pietrinferni, A., Piotto, G., Milone, A. P., Bedin, L. R., \& Anderson, J. 2008, ApJ, 672, L115

D’Antona, F., Bellazzini, M., Caloi, V., Pecci, F. F., Galleti, S., \& Rood, R. T. 2005, ApJ, 631, 868

D’Antona, F., \& Caloi, V. 2004, ApJ, 611, 871

D'Antona, F., \& Ventura, P. 2008, Messenger, 134, 18

de Laverny, P., \& Gustafsson, B. 1998, A\&A, 332, 661

Decressin, T., Meynet, G., Charbonnel, C., Prantzos, N., \& Ekström, S. 2007, A\&A, 464, 1029
D’Ercole, A., Vesperini, E., D’Antona, F., McMillan, S. L. W., \& Recchi, S. 2008, MNRAS, 391, 825

Fenner, Y., Campbell, S., Karakas, A. I., Lattanzio, J. C., \& Gibson, B. K. 2004, MNRAS, 353, 789

Gratton, R., Sneden, C., \& Carretta, E. 2004, ARA\&A, 42, 385

Grevesse, N., \& Sauval, A. J. 1998, Space Sci. Rev., 85, 161

Grundahl, F., Catelan, M., Landsman, W. B., Stetson, P. B., \& Andersen, M. I. 1999, ApJ, 524, 242

Izzard, R. G., Lugaro, M., Karakas, A. I., Iliadis, C., \& van Raai, M. 2007, A\&A, 466, 641

Karakas, A. I., Fenner, Y., Sills, A., Campbell, S. W., \& Lattanzio, J. C. 2006a, ApJ, 652, 1240

Karakas, A., \& Lattanzio, J. C. 2007, PASA, 24, 103

Karakas, A. I., Lugaro, M. A., Wiescher, M., Görres, J., \& Ugalde, C. 2006b, ApJ, 643, 471

Lee, Y.-W., Gim, H. B., \& Casetti-Dinescu, D. I. 2007, ApJ, 661, L49

Lugaro, M., Ugalde, C., Karakas, A. I., Görres, J., Wiescher, M., Lattanzio, J. C., \& Cannon, R. C. 2004, ApJ, 615, 934

Mackey, A. D., Broby Nielsen, P., Ferguson, A. M. N., \& Richardson, J. C. 2008, ApJ, 681, L17

Milone, A. P., et al. 2008, ApJ, 673, 241

Norris, J. E. 2004, ApJ, 612, L25

Norris, J. E., \& Da Costa, G. S. 1995, ApJ, 447, 680

Piotto, G., et al. 2005, ApJ, 621, 777

Plez, B., Masseron, T., \& Van Eck, S. 2008, in ASP Conf. Ser., Cool Stars, Stellar Systems and the Sun, in press

Pumo, M. L., D’Antona, F., \& Ventura, P. 2008, ApJ, 672, L25

Reddy, B. E., Lambert, D. L., Gonzalez, G., \& Yong, D. 2002, ApJ, 564, 482

Renzini, A. 2008, MNRAS, 391, 354

Renzini, A., \& Buzzoni, A. 1986, in Astrophysics and Space Science Library 122, Spectral Evolution of Galaxies, ed. C. Chiosi \& A. Renzini (Dordrecht: D. Reidel Publishing Co.), 195

Salaris, M., Cassisi, S., \& Pietrinferni, A. 2008, ApJ, 678, L25

Smith, V. V., Cunha, K., Ivans, I. I., Lattanzio, J. C., Campbell, S., \& Hinkle, K. H. 2005, ApJ, 633, 392

Sneden, C. 1973, ApJ, 184, 839

Sollima, A., Ferraro, F. R., Pancino, E., \& Bellazzini, M. 2005, MNRAS, 357 265

Stetson, P. B. 1981, AJ, 86, 687

Ventura, P., \& D'Antona, F. 2005a, A\&A, 431, 279

Ventura, P., \& D'Antona, F. 2005b, A\&A, 439, 1075

Ventura, P., \& D'Antona, F. 2006, A\&A, 457, 995

Villanova, S., et al. 2007, ApJ, 663, 296

Yong, D., Aoki, W., Lambert, D. L., \& Paulson, D. B. 2006, ApJ, 639, 918

Yong, D., \& Grundahl, F. 2008, ApJ, 672, L29

Yong, D., Grundahl, F., Johnson, J. A., \& Asplund, M. 2008a, ApJ, 684, 1159

Yong, D., Melendez, J., Cunha, K., Karakas, A. I., Norris, J. E., \& Smith, V. V. 2008b, ApJ, 689, 1020 